\title{
A Potential Mechanism of Anticancer Immune Response Coincident With Immune-related Adverse Events in Patients With Renal Cell Carcinoma
}

\author{
TAIGO KATO $^{1,2}$, EISUKE TOMIYAMA ${ }^{1}$, YOKO KOH $^{1}$, MAKOTO MATSUSHITA $^{1}$, YUJIRO HAYASHI ${ }^{1}$, \\ KOSUKE NAKANO $^{1}$, YU ISHIZUYA ${ }^{1}$, CONG WANG $^{1}$, KOJI HATANO ${ }^{1}$, ATSUNARI KAWASHIMA ${ }^{1}$, \\ TAKESHI UJIKE ${ }^{1}$, KEISUKE KAWASAKI ${ }^{3}$, EIICHI MORII ${ }^{3}$, KUNIHITO GOTOH ${ }^{4}$, HIDETOSHI EGUCHI ${ }^{4}$, \\ KAZUMA KIYOTANI ${ }^{5}$, KAZUTOSHI FUJITA ${ }^{1}$, NORIO NONOMURA ${ }^{1}$ and MOTOHIDE UEMURA ${ }^{1,2}$ \\ ${ }^{1}$ Department of Urology, Osaka University Graduate School of Medicine, Osaka, Japan; \\ ${ }^{2}$ Department of Urological Immuno-oncology, Osaka University Graduate School of Medicine, Osaka, Japan; \\ ${ }^{3}$ Department of Pathology, Osaka University Graduate School of Medicine, Osaka, Japan; \\ ${ }^{4}$ Department of Gastroenterological Surgery, Osaka University Graduate School of Medicine, Osaka, Japan; \\ ${ }^{5}$ Cancer Precision Medicine Center, Japanese Foundation for Cancer Research, Tokyo, Japan
}

\begin{abstract}
Background/Aim: Some reports showed encouraging efficacy of immune checkpoint inhibitors among patients who experienced immune-related adverse events (irAEs). Thus, characterization of T-cell repertoire and immune signatures in peripheral blood mononuclear cells (PBMCs) and tumors before and after immune checkpoint inhibitors treatment should contribute to better understanding of irAE-provoked anticancer immune responses. Materials and Methods: We applied expression analysis of immune-related genes and T-cell receptor sequencing in tumor and PBMCs from five patients with renal cell carcinoma before combined immunotherapy and at the onset of severe irAEs. Results: We found that the cluster of differentiation 8 (CD8)/forkhead box P3(FOXP3), granzyme $B(G Z M B) / C D 3$, perforin $1(P R F 1) / C D 3$ and programmed cell death $1(P D 1) / C D 8$ expression ratios were significantly elevated in PBMCs at the onset of irAEs. In addition, we found expansion of certain T-cell clones in metastatic tissue after irAEs, which were already increased in peripheral blood at the onset of irAEs. Conclusion: irAE-
\end{abstract}

This article is freely accessible online.

Correspondence to: Taigo Kato (ORCID ID: 0000-0002-86811407), MD, Ph.D., Department of Urology, Osaka University Graduate School of Medicine, 2-2 Yamadaoka, Suita, Osaka, 5650871, Japan. Tel: +81 668793531, Fax: +81 668793534, e-mail: kato@uro.med.oaska-u.ac.jp

Key Words: Immune checkpoint inhibitors, immune-related adverse events (irAEs), T-cell receptor, renal cell carcinoma, nextgeneration sequencing, immune response. provoked T-cells may also circulate and attack distant tumors, leading to durable response in patients with irAEs.

Immune checkpoint inhibitors (ICIs) have revolutionized the field of cancer therapy in recent years and remarkably extended the prognosis associated with several cancer types (1). Patients can face discontinuation of ICIs due to severe and sometime life-threatening immune-related adverse events (irAEs) (2). The combination of inhibitors of programmed cell death 1 (PD1) and cytotoxic T-lymphocyte antigen 4 (CTLA4), namely nivolumab and ipilimumab, was shown to increase the incidence of irAEs compared to that each monotherapy despite the high response rate (3). At the same time, it was reported that the response rate and survival did not differ between patients with advanced melanoma who did and did not discontinue because of irAEs (CheckMate 069 and 067 studies) $(4,5)$. These findings led to the hypothesis that patients with irAEs may not only have a strong autoimmune response but also anticancer response as a result of reinvigoration of exhausted Tcells with ICIs. The immune mechanisms of durable clinical response after developing irAEs remain unclear.

T-Cell receptors (TCR), most of which consist of a heterodimer of TCR alpha (TCRA) and beta (TCRB) chains, are expressed on the surface of T-cells. In order to recognize a huge variety of antigens, TCR genes undergo a somatic V(D)-J recombination process that generates a huge diverse repertoire of T-cells. Through next-generation sequencingbased TCR analysis using mRNAs, several reports expanded perceptions on T-cell immunity, showing it to play pivotal roles in various types of cancer (6-11).

In the present study, we performed expression analysis of immune-related genes using peripheral blood of patients with 
Table I. List of TaqMan probes designed for real-time polymerase chain reaction.

\begin{tabular}{lcc}
\hline Marker & Gene symbol & Assay ID \\
\hline Cluster of differentiation 3 (CD3) & CD3E & Hs01062241_m1 \\
Forkhead box P3 & FOXP3 & Hs01085834_m1 \\
Cluster of differentiation 4 (CD4) & Hs01058407_m1 \\
Cluster of differentiation 8 (CD8) & CD 4 & Hs002335520_m1 \\
Granzyme B & GZMB & Hs00188051_m1 \\
Perforin 1 & PRF1 & Hs00175473_m1 \\
Cytotoxic T-lymphocyte associated protein 4 (CTLA4) & CTLA4 & Hs01550088_m1 \\
Programmed cell death 1 (PD1) & PDCD1 & Hs00969556_m1 \\
Cluster of differentiation 39 (CD39) & ENTPD1 & Hs01025372_m1 \\
Cluster of differentiation 103 (CD103) & ITGAE & Hs00195153_m1 \\
Killer cell lectin-like receptor G1 & KLRG1 & Hs00958618_m1 \\
T-Cell immunoglobulin and mucin domain-containing protein 3(TIM3) & HAVCR2 & Hs02758991_g1 \\
Glyceraldehyde 3-phosphate dehydrogenase & GAPDH & \\
\hline
\end{tabular}

renal cell carcinoma (RCC) who discontinued combination therapy of nivolumab and ipilimumab because of severe irAEs. In addition, we applied next-generation sequencing approach to characterize TCR repertoires in metastatic tumor tissues with significant regression after discontinuation of combination therapy. Our results showed that TCR repertoire analysis and immune-profiling of irAE-experienced cancer patients may lead to a better understanding of the irAErelated anticancer immune response.

\section{Materials and Methods}

Patient population. Eligible patients had stage IV histologically documented RCC who had received no prior treatment. Between September 2018 and September 2019, a total of five patients were enrolled and received combination therapy of nivolumab and ipilimumab. All patients were confirmed to have RCC by nephrectomy or needle biopsy and had metastases. Follow-up was according to the institutional standard for advanced RCC, with clinical examination and laboratory testing every 3 months and analysis by computed tomography scan every 3 months. The study protocol was approved by the Institutional Review Board of Osaka University (approval number 668-5). All patients provided written informed consent.

In this study, irAE was defined as an adverse event which had a certain or probable causal association with the use of PD1 or CTLA4 inhibitor as assessed on the World Health Organization Uppsala Monitoring Centre scale (12). All irAEs were reported using the National Cancer Institute Common Terminology Criteria for Adverse Events, version 5.0 (13).

To characterize changes of systemic immune signature at the incidence of irAEs, we obtained peripheral blood mononuclear cell (PBMC) samples before starting the combination therapy, and at the onset of irAEs. The control group was set as patients who had responses to the combination therapy but without experiencing irAEs; from these patients, we also collected PBMC samples before the combination therapy and at 3 months after the therapy. In addition, we obtained pancreatic metastatic tissue sample from a patient who underwent pancreatectomy after discontinuation of combination therapy.
Gene-expression analysis. Total RNA from metastatic tumor tissues and PBMCs were isolated using RNAeasy Mini kit (Qiagen, Valencia, CA, USA). Complementary DNA (cDNA) was synthesized from total RNA using Prime Script RT reagent kit (Takara Bio Inc., Shiga, Japan). Real-time PCR was performed using TaqMan gene expression assay in the QuantStudio 5 system, according to the manufacturer's instructions (ThermoFisher, Waltham, MA, USA). We examined transcriptional levels of 12 genes [cluster of differentiation $3(C D 3), C D 8, C D 4$, forkhead box P3 (FOXP3), granzyme B (GZMB), perforin 1 (PRF1), CD39, $C D 103$, killer cell lectin-like receptor G1 (KLRG1), PD1, CTLA4, and T-cell immunoglobulin and mucin domain-containing protein 3 (TIM3)] associated with cytotoxic T-cells, effector T-cells, and exhausted T-cells in PBMCs. The expression levels were normalized with that of glyceraldehyde 3-phosphate dehydrogenase (GAPDH). We then evaluated expression relative to that of T-cell markers CD3, CD4 and CD8. All TaqMan probes are listed in Table I.

TCR sequencing. Sequencing libraries of TCR were prepared as described previously $(6,7)$ and subjected to sequencing on the Illumina Miseq platform, using 600 cycles Miseq Reagent Kit V3 (Illumina, San Diego, CA, USA).

Qualitative immunohistochemical studies. Paraffin-embedded tissues were cut into 5- $\mu \mathrm{m}$-thick sections and tissue slides were stained on Ventana Benchmark Ultra System (Roche, Basel, Switzerland). Antibody to human CD8 (clone SP57; Roche) or human CD4 (clone SP35; Roche) was used as a primary antibody and antigen-antibody binding was visualized by Optiview DAB IHC Detection Kit (Roche) according to the manufacturer's protocols.

Statistical analysis. The Mann-Whitney $U$-test (two-tailed) was performed for comparison of gene-expression levels before the combination therapy and at the onset of irAEs using JMP Pro 14.0.0 (SAS Institute Inc., Cary, NC, USA). To analyze the changes of complementarity determining region 3 (CDR3) clonotypes in PBMCs, we focused on a total of 44 abundant CDR3 clonotypes $(\geq 1 \%$ frequency among total TCRA and TCRB reads) which were expanded in the pancreatic metastasis. Then, we applied Fisher's exact test to examine the significance in the increase of each of these CDR3 


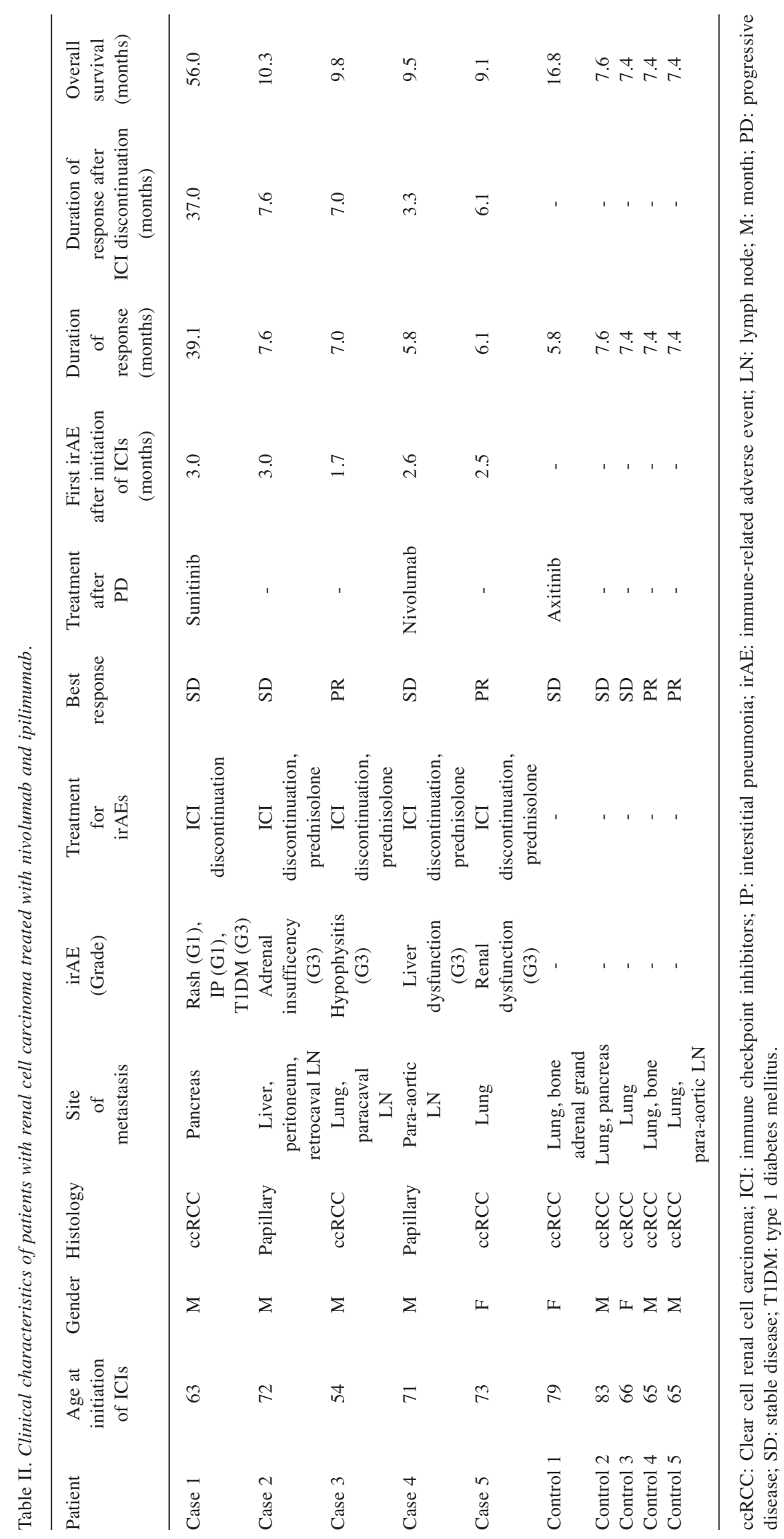


clonotypes in PBMC samples. In this analysis, significance was set at $p<0.0011$ ( 0.05 divided by 44$)$ according to Bonferroni correction. In other analyses, differences were considered to be significant at $p<0.05$.

\section{Results}

Clinical characteristics of patients with cancer. The clinical information of five patients with RCC is shown in Table II. The median age of the patients was 71 years (range $=54-73$ years). All patients received nivolumab and ipilimumab as the first-line therapy. All patients experienced irAEs of grade 3, which occurred within 3 months after initiation of the combination therapy. irAEs gradually improved after discontinuation of the combination therapy and administration of corticosteroid. Best clinical responses as evaluated by the Response Evaluation Criteria in Solid Tumors version 1.1 (14) were partial response in two patients and stable disease in the remaining three. Four out of five patients responded for at least 6 months without any additional therapy.

Expression of immune-related genes in peripheral blood before combination therapy and at the onset of irAEs. To characterize changes of the systemic immune signature at the occurrence of irAEs, we obtained PBMC samples before starting combination therapy of nivolumab and ipilimumab, and at the onset of irAEs. For the control group, we also collected PBMC samples before the combination therapy and at 3 months after the therapy. We examined transcriptional levels of 12 genes associated with cytotoxic T-cells, effector T-cells, and exhausted T-cells in peripheral bIood with realtime PCR (Figure 1A). In PBMCs at the onset of irAEs, we found the CD8/FOXP3, CD8/CD4, GZMB/CD3, and $P R F 1 / C D 3$ expression ratios were significantly increased when compared with those of patients without irAEs (Figure 1B), implying that cytolytic $\mathrm{CD}^{+} \mathrm{T}$-cells were strongly expanded due to irAEs. Interestingly, $P D 1 / C D 3$ and $P D 1 / C D 8$ expression ratios were also increased with irAEs (Figure 1B), whereas that of PD1/CD4 was not (data not shown). These results suggest the development of irAEs was closely correlated with systemic activation of $\mathrm{CD} 8^{+} \mathrm{PD} 1^{+} \mathrm{T}-$ cells, which are probably activated by recognizing cancerspecific antigens in tumors $(15,16)$.

Drastic increase of $\mathrm{CD}^{+}$and $C D 4^{+} \mathrm{T}$-cells in metastasis tissue of patients with severe irAEs. Among the five cases with irAEs, one case underwent pancreatectomy for pancreatic metastasis and maintained stable disease status for 2 years after the discontinuation of the combination therapy due to severe irAEs (Figure 2). Firstly, we examined the status of T-cell infiltration in the pathological specimen. Through immunohistochemical analysis of CD8 and CD4 on the surgically resected pancreatic metastasis tissue, we confirmed the number of infiltrating $\mathrm{CD}^{+}$and $\mathrm{CD} 4^{+}$T-cells in the tumor as being significantly higher compared to normal pancreatic tissue (Figure 3), in which $\mathrm{CD}^{+}$and $\mathrm{CD} 4^{+} \mathrm{T}$-cells were rarely present.

Expanded T-cells in metastatic tissue are also present in the systemic circulation at the onset of irAEs. Next, to examine the effect of the combination therapy on the T-cell repertoire in the pancreatic metastasis, we performed TCR sequencing for the metastatic tissue of this case. Through cDNA sequencing of TCRs, we obtained total sequence reads of 525,084 and 504,965 mapped to V, (D), J, and C segments for TCRA and TCRB, respectively. From these TCR reads, we identified 10,220 and 21,020 unique CDR3 clonotypes for TCRA and TCRB, respectively. After sorting out CDR3 clonotypes according to their frequencies in the metastatic tumor tissue, we determined the proportions of abundant CDR3 clonotypes, which were defined as the clonotypes with the frequency of $1 \%$ or higher, and found an oligoclonal expansion of T-cells in the metastatic tissue (Figure 4A). Interestingly, only a few TCRA and TCRB CDR3 clonotypes among abundant T-cell clonotypes (frequency of $\geq 0.5 \%$ ) were shared between in pancreatic metastasis and in normal pancreatic tissue (Figure $4 \mathrm{~B}$ and $\mathrm{C}$ ). We further examined the frequencies of TCRA and TCRB CDR3 clonotypes which were enriched in the pancreatic metastasis tissue in a peripheral blood sample. As a result, several TCRA and TCRB CDR3 clonotypes expanded in the tissue were found to be significantly increased in PBMCs at the onset of skin rash after combination therapy compared with the pre-treatment sample of PBMCs $(p<0.0011$, Figure 5). These findings may indicate that a robust systemic immune reaction inducing irAEs might also unleash cancer antigenspecific T-cells, which circulate systemically and attack cancer cells in distant tumoral lesions.

\section{Discussion}

Immunotherapy targeting immune checkpoint molecules is now the most attractive therapy in the field of cancer (17). Despite the high response rate, it is undeniable that a certain population of patients are forced to discontinue ICIs because of severe irAEs (2). On the other hand, prior studies have shown encouraging clinical outcomes even for patients who discontinued ICIs, implying that the experience of an irAE may also induce cancer-reactive T-cells $(4,5,18)$. However, the molecular mechanism of anticancer immune reaction related to irAEs is still largely unknown. In this study, we performed expression analysis of immune-related genes in peripheral blood in patients with and without irAEs. Moreover, we also preformed TCR repertoire profiling in metastatic tumor tissue from a patient with a sustained durable response after discontinuation of ICIs to investigate whether irAEs might be a manifestation that anticancer immune reaction had been activated.

Through analysis of the TCR repertoire and immune-related gene expression, we have demonstrated several novel findings 


\section{A}

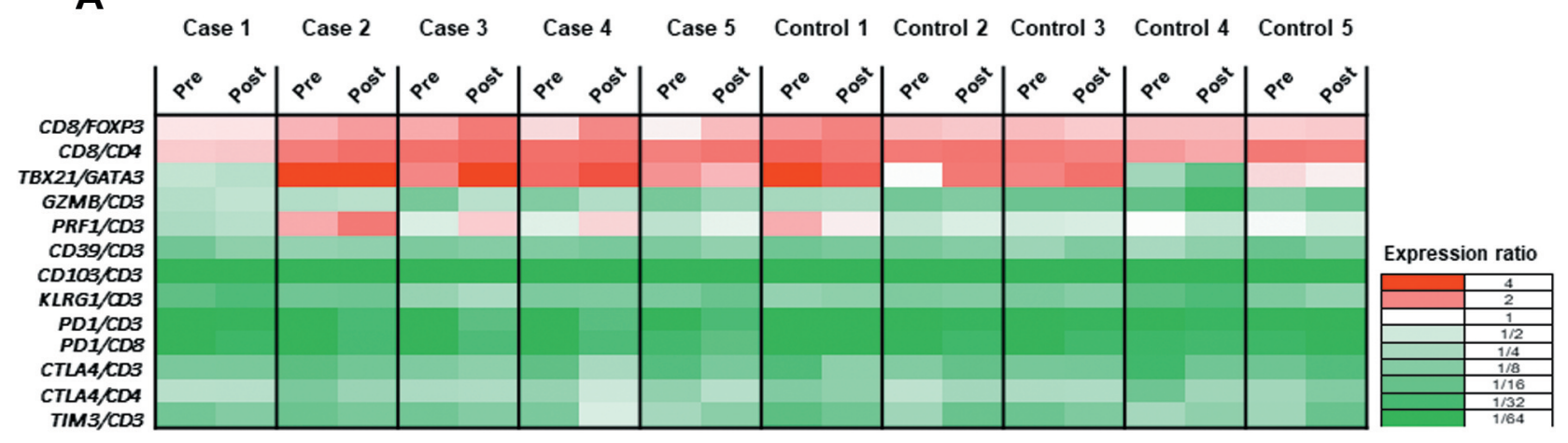

B
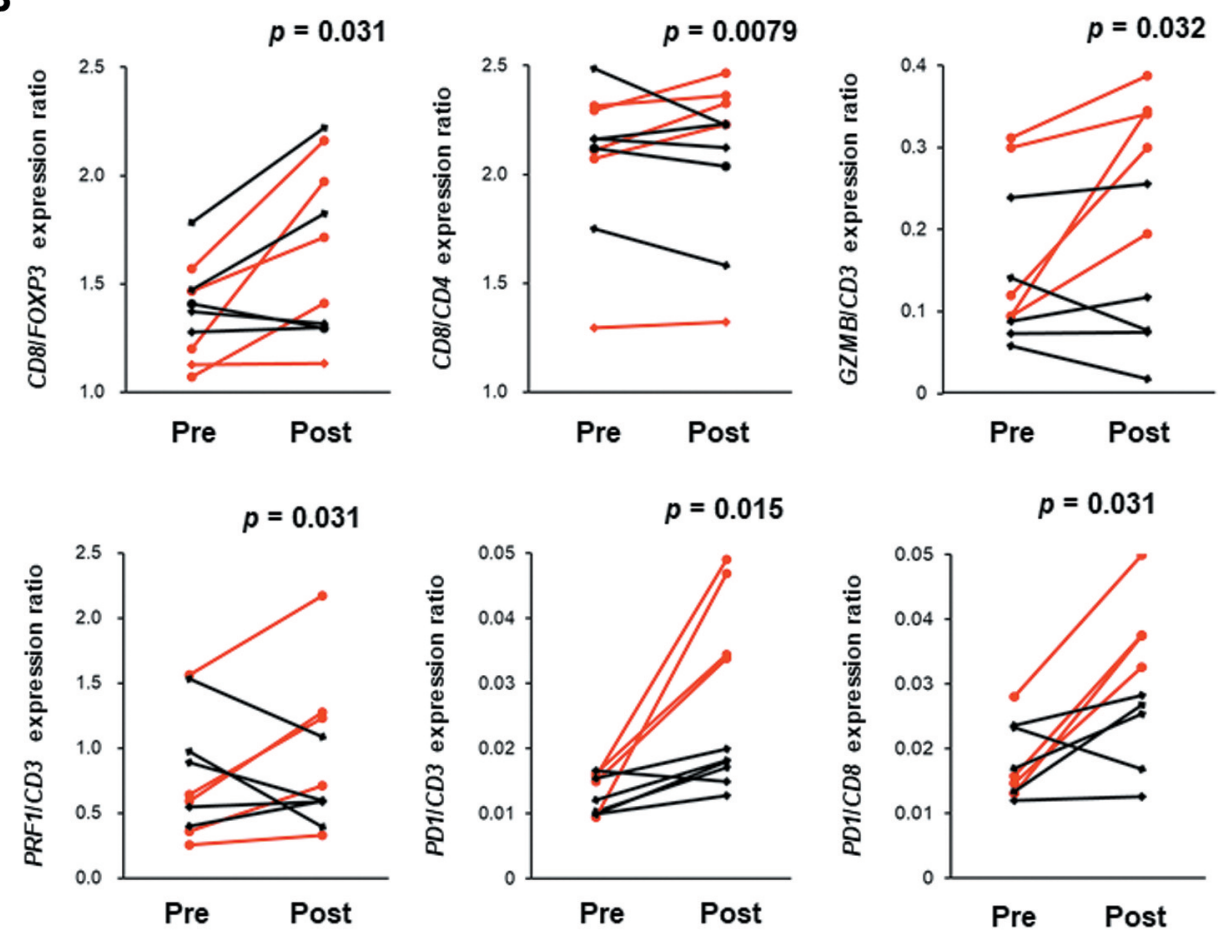

Figure 1. Expression of immune-related genes in blood samples before combination therapy and at the onset of immune-related adverse events (irAEs). A: Heatmap showing the ratios of immune-related gene expression in the peripheral blood mononuclear cells (PBMCs) of pre-combination therapy (pre) and at the onset of irAEs (post). In the control group, post indicates the results for PBMC samples at 3 months after the initiation of the combination therapy. The expression level of each gene was calculated relative to that of glyceraldehyde 3-phosphate dehydrogenase (GAPDH). $B$ : Line graphs showing the comparative analysis of ratio of immune-related gene expression in pre-treatment (pre) and post-treatment (post). Red and black symbols indicate tumor samples from patients with and without irAEs, respectively.

that may explain irAE-related anticancer immune responses. Firstly, we found that expression levels of cytolytic markers on CD ${ }^{+}$T-cells, namely GZMB and PRF1, were significantly increased in peripheral blood at the onset of irAEs. It is well known that $\mathrm{CD}^{+}$cytotoxic T-cells play a central role in cancer immunosurveillance and may attack normal organs, which cause the damage in autoimmune reactions (19). In addition, $\mathrm{CD}^{+}$cytotoxic T-cells predominantly circulate over Treg cells in peripheral blood as evidenced by the increase of the CD8/FOXP3 expression ratio at the onset of irAEs. Moreover, patients with irAEs had a significantly increased number of $\mathrm{CD}^{+}{ }^{+} \mathrm{PD} 1^{+}$cells, which are thought to recognize cancerassociated antigens $(15,16)$. These results indicate that the combination of ICIs may induce not only the expansion of autoreactive cytotoxic T-lymphocytes which cause adverse events in normal tissues, but also cancer-antigen specific T-cells. 


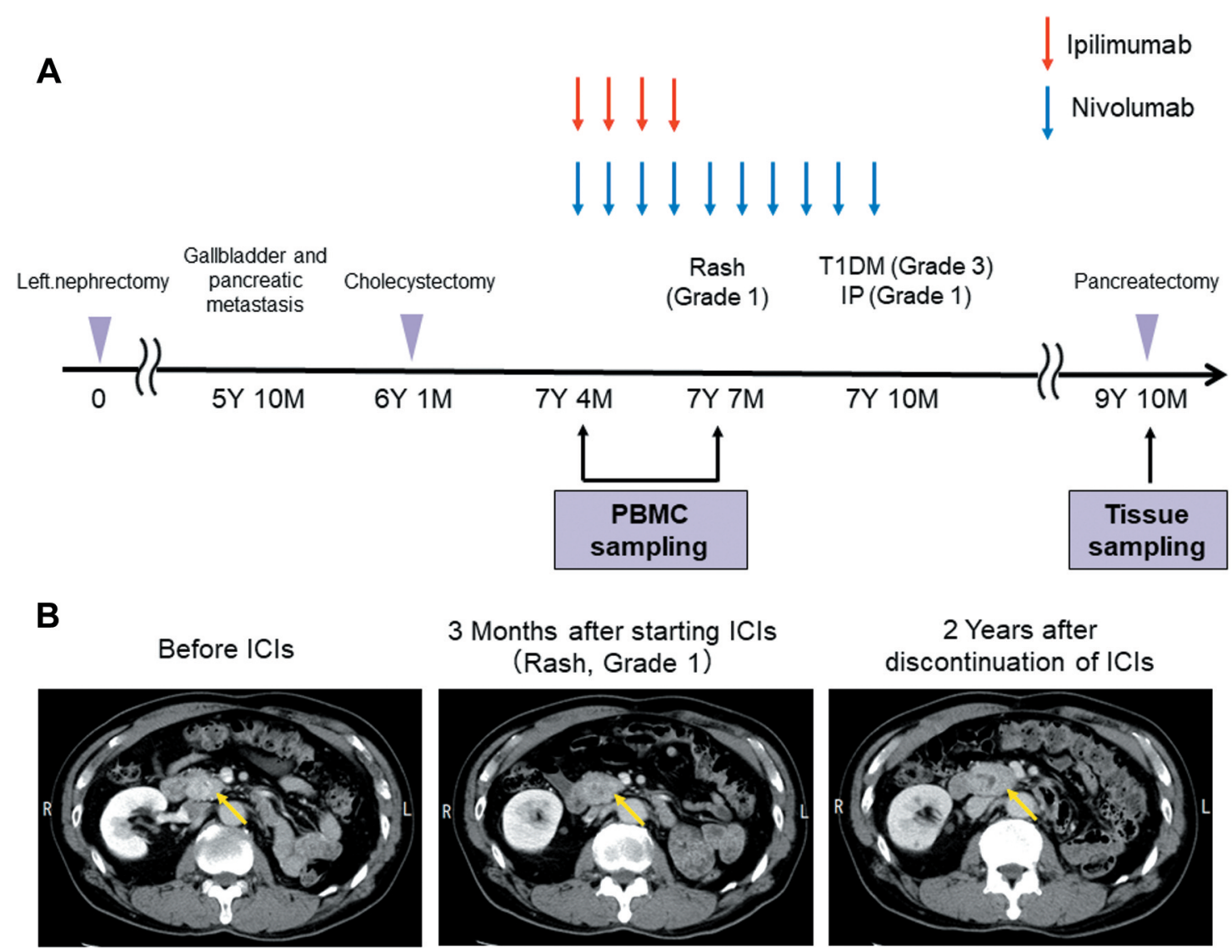

Figure 2. Clinical course of case 1 with rash, interstitial pneumonia, and type 1 diabetes induced by nivolumab and ipilimumab. A: After discontinuation of the combination therapy because of several immune-related adverse events (grade 1 rash, grade 1 interstitial pneumonia and grade 3 type 1 diabetes), the patient sustained a durable response for 2 years and underwent pancreatectomy for a single metastasis. B: Serial computed tomographic scans are shown before treatment with immune checkpoint inhibitor (ICI), at the onset of skin rash 3 months after starting this therapy, and 2 years after discontinuation of ICIs. Y: Years; M: months; IP: interstitial pneumonia; TIDM: type 1 diabetes mellitus.

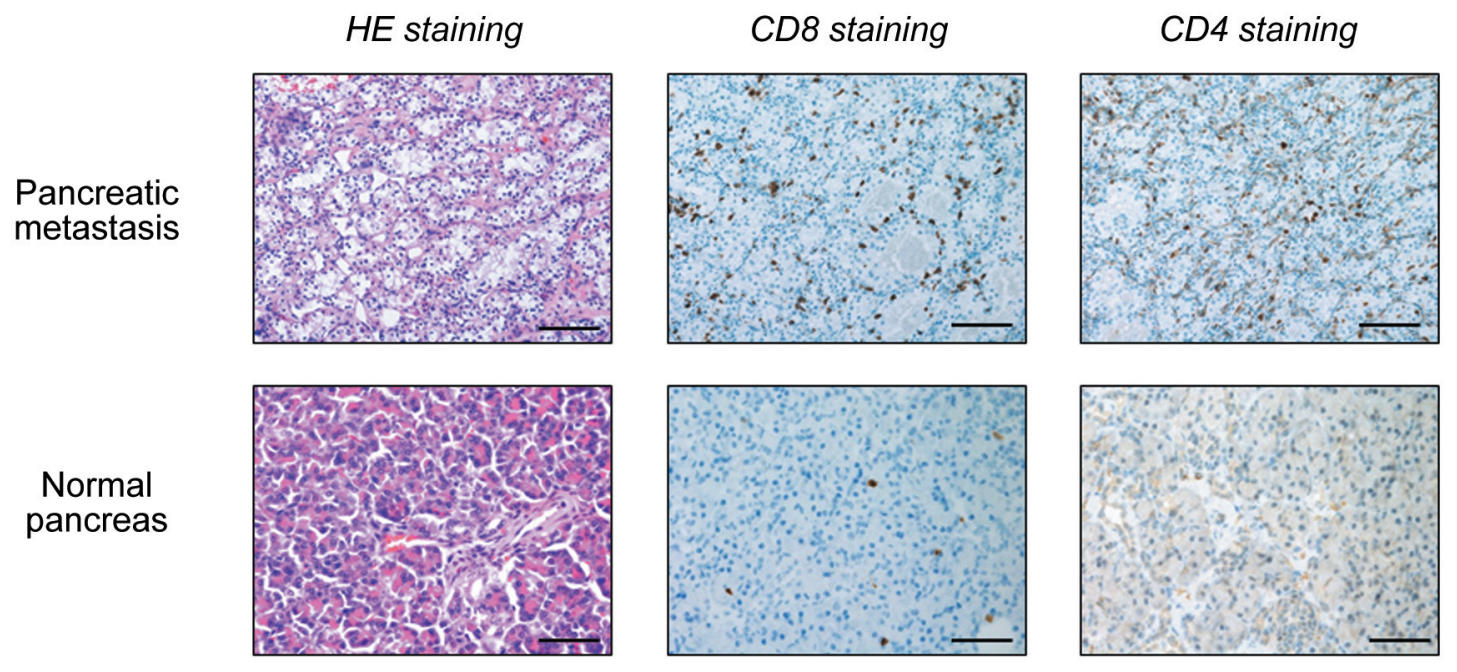

Figure 3. Infiltration of $\mathrm{CD}^{+}$and $C D 8^{+}$T-cells into pancreatic metastasis tissue of case 1. Representative immunohistochemistry for hematoxylineosin (HE), CD4 and CD8 staining in pancreatic metastasis of case 1. Scale bars $=200 \mu \mathrm{m}$. 
A
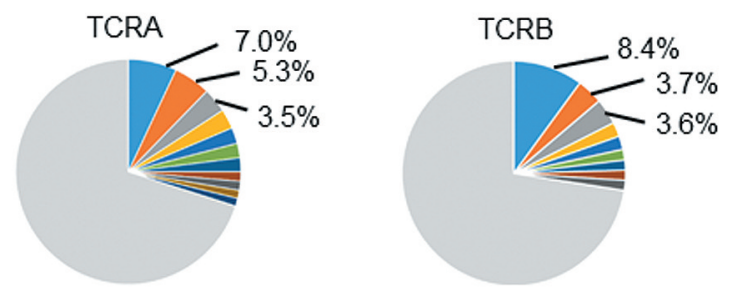

B

\section{C}
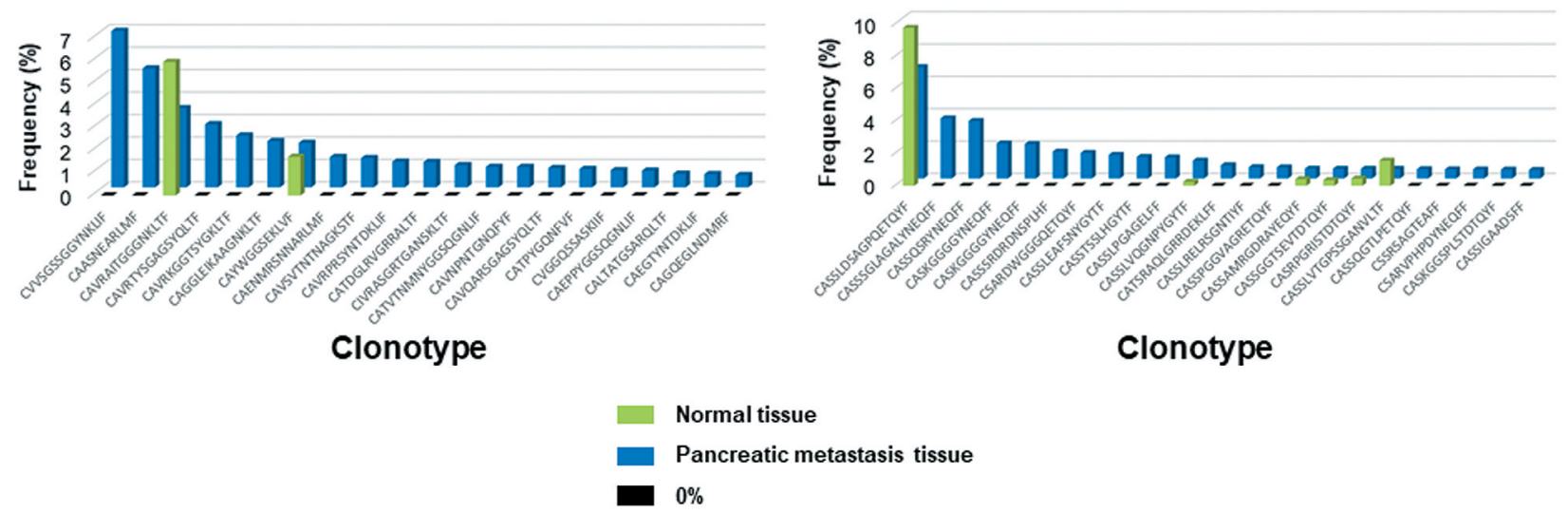

Figure 4. Combination therapy induced clonal T-cell expansion in pancreatic metastasis. A: T-cell receptors (TCR) sequencing revealed clonal Tcell expansion in pancreatic metastasis. The distribution of the abundant TCR alpha (TCRA) and beta (TCRB) complementarity determining region 3 (CDR3) clonotypes with the read frequency of $1 \%$ or higher is presented in pie charts. Gray indicates the proportion of CDR 3 clonotypes with a read frequency below 1\%. B: Frequency distribution of TCRA clonotypes. C: Frequency distribution of TCRB clonotypes. Only two TCRA and five $T C R B$ CDR3 clonotypes were shared in pancreatic metastasis and normal pancreas among most abundant clonotypes (frequency of $\geq 0.5 \%$ ) observed in the metastasis.

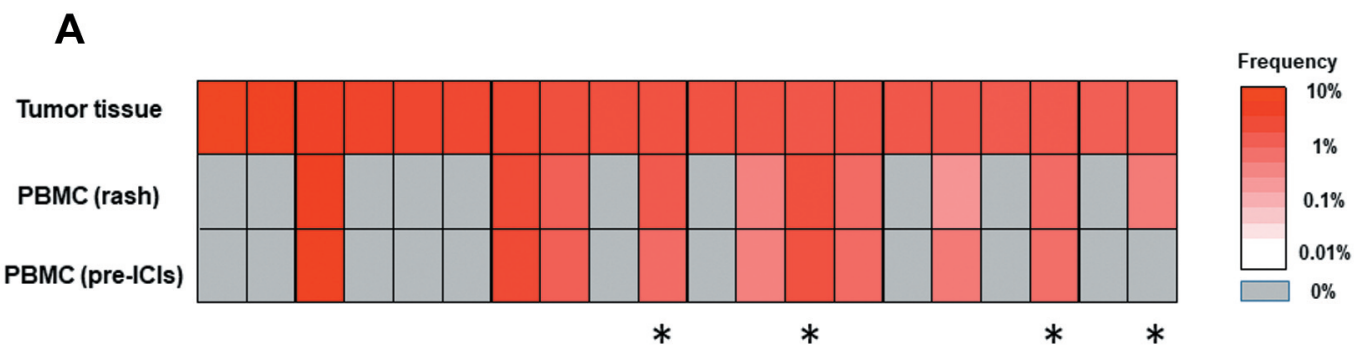

\section{B}

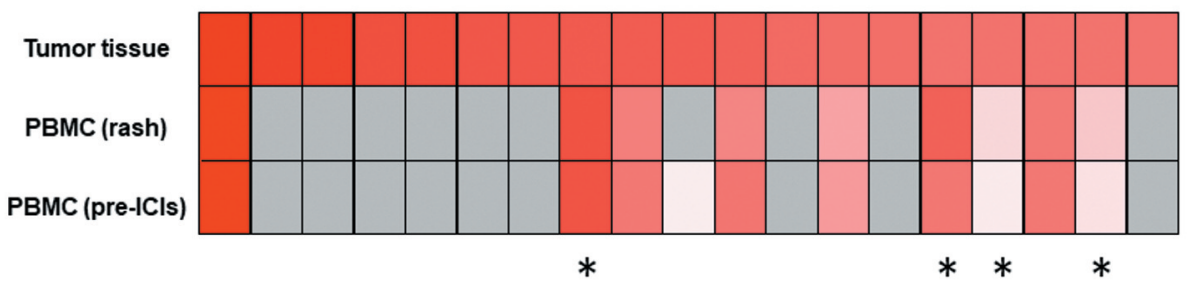

Figure 5. Expanded T-cell clones in pancreatic metastasis were already present and increased in peripheral blood at the onset of immune-related adverse events (irAEs). Heatmaps showing the distribution of TCR alpha (TCRA) (A) and beta (TCRB) complementarity determining region 3 (CDR3) clonotypes $(B)$ in peripheral blood mononuclear cell (PBMC) samples of case 1. The top 20 CDR3 clonotypes of pancreatic metastasis tissue were selected. The asterisks indicate CDR3 clonotypes which were significantly increased at the onset of irAEs compared with these before combination therapy with (pre-ICIs) ( $p<0.011$ to correct multiple testing). 
Secondly, we found that a small subset of T-cells was significantly expanded in metastatic tumor tissue after irAEs. In addition, immunohistochemical analysis of CD8 and CD4 also revealed the significant increase of infiltrating $\mathrm{CD}^{+}$and $\mathrm{CD}^{+}{ }^{-} \mathrm{T}$-cells in the tumor tissue, suggesting that the combination of ICI activated T-lymphocytes that possibly recognize cancer-specific antigens in tumor tissue. Interestingly, some of these T-cell clones were also increased in peripheral blood at the onset of irAEs, indicating that some clonally-expanded T-cells with irAEs can circulate systemically in the body and might attack cancer cells in metastatic lesions. These findings may partly explain the mechanism underlying why a certain population of patients experiencing irAEs can maintain the regression of tumor legions and achieve a favorable clinical response even after discontinuing ICI therapy.

In summary, our results provide the first evidence that combination therapy of CTLA4 and PD1 blockade induces strong systemic immune/autoimmune responses associated with infiltration of $\mathrm{CD}^{+}$and $\mathrm{CD} 4^{+}$T-cells and oligoclonal expansion of possible antitumor T-cells. For translation into the clinic, unique expanded TCR sequences identified in peripheral blood at onset of irAEs in individual patients might have the potential to predict subsequent clinical outcome.

\section{Conflicts of Interest}

The Authors declare no competing financial interests.

\section{Authors' Contributions}

T.K. planned the entire project, performed data analysis, and drafted the article; M.U. planned, supervised the entire project and completed the article; N.N. provided the study design and the working hypothesis and completed the article; K.K. designed the study, conducted experiments, performed data analysis, and completed the article; T.I. conducted experiments and performed data analysis; E.T., Y.K., M.M., Y.H., K.N., Y.I., C.W., Y.Y., K.H., A.K., T.U., A.N., and K.F. conducted data analysis and provided scientific advice.

\section{Acknowledgements}

The Authors thank Atsuko Yasumoto and Mutsumi Tsuchiya for sample processing and data collection, Saki Matsumoto for technical assistance, and Drs. Rui Yamaguchi and Satoru Miyano at the University of Tokyo for developing the algorithm of TCR repertoire analysis and helpful support in data management. The supercomputing resource (http://sc.hgc.jp/shirokane.html) was provided by the Human Genome Center, the Institute of Medical Science, The University of Tokyo. This work was also supported by JSPS KAKENHI (Grant-in-Aid for Scientific Research (C), grant numbers 18K09133, Grant-in-Aid for Scientific Research (B), grant numbers 17H04329) and ONO PHARMACEUTICAL CO., LTD.

\section{References}

1 Havel JJ, Chowell D and Chan TA: The evolving landscape of biomarkers for checkpoint inhibitor immunotherapy. Nat Rev Cancer 19(3): 133-150, 2019. PMID: 30755690. DOI: 10.1038/s41568-019-0116-x

2 Postow MA, Sidlow R and Hellmann MD: Immune-related adverse events associated with immune checkpoint blockade. N Engl J Med 378(2): 158-168, 2018. PMID: 29320654. DOI: 10.1056/NEJMra1703481

3 Rotte A: Combination of CTLA-4 and PD-1 blockers for treatment of cancer. J Exp Clin Cancer Res 38(1): 255, 2019. PMID: 31196207. DOI: 10.1186/s13046-019-1259-z

4 Hodi FS, Chiarion-Sileni V, Gonzalez R, Grob JJ, Rutkowski P, Cowey CL, Lao CD, Schadendorf D, Wagstaff J, Dummer R, Ferrucci PF, Smylie M, Hill A, Hogg D, Marquez-Rodas I, Jiang J, Rizzo J, Larkin J and Wolchok JD: Nivolumab plus ipilimumab or nivolumab alone versus ipilimumab alone in advanced melanoma (CheckMate 067): 4-Year outcomes of a multicentre, randomised, phase 3 trial. Lancet Oncol 19(11): 1480-1492, 2018. PMID: 30361170. DOI: 10.1016/S14702045(18)30700-9

5 Regan MM, Werner L, Rao S, Gupte-Singh K, Hodi FS, Kirkwood JM, Kluger HM, Larkin J, Postow MA, Ritchings C, Sznol M, Tarhini AA, Wolchok JD, Atkins MB and McDermott DF: Treatment-free survival: A novel outcome measure of the effects of immune checkpoint inhibition - a pooled analysis of patients with advanced melanoma. J Clin Oncol 37(35): 33503358, 2019. PMID: 31498030. DOI: 10.1200/JCO.19.00345

6 Kato T, Iwasaki T, Uemura M, Nagahara A, Higashihara H, Osuga K, Ikeda Y, Kiyotani K, Park JH, Nonomura N and Nakamura Y: Characterization of the cryoablation-induced immune response in kidney cancer patients. Oncoimmunology 6(7): e1326441, 2017. PMID: 28811963. DOI: 10.1080/2162402X.2017.1326441

7 Kato T, Park JH, Kiyotani K, Ikeda Y, Miyoshi Y and Nakamura Y: Integrated analysis of somatic mutations and immune microenvironment of multiple regions in breast cancers. Oncotarget 8(37): 62029-62038, 2017. PMID: 28977923. DOI: 10.18632/oncotarget. 18790

8 Inoue H, Park JH, Kiyotani K, Zewde M, Miyashita A, Jinnin M, Kiniwa $Y$, Okuyama R, Tanaka R, Fujisawa $Y$, Kato $H$, Morita A, Asai J, Katoh N, Yokota K, Akiyama M, Ihn H, Fukushima $S$ and Nakamura Y: Intratumoral expression levels of PD-L1, GZMA, and HLA-A along with oligoclonal T-cell expansion associate with response to nivolumab in metastatic melanoma. Oncoimmunology 5(9): e1204507, 2016. PMID: 27757299. DOI: 10.1080/2162402X.2016.1204507

9 Tamura K, Hazama S, Yamaguchi R, Imoto S, Takenouchi H, Inoue Y, Kanekiyo S, Shindo Y, Miyano S, Nakamura Y and Kiyotani K: Characterization of the T-cell repertoire by deep Tcell receptor sequencing in tissues and blood from patients with advanced colorectal cancer. Oncol Lett 11(6): 3643-3649, 2016. PMID: 27284367. DOI: 10.3892/ol.2016.4465

10 Matsuda T, Miyauchi E, Hsu YW, Nagayama S, Kiyotani K, Zewde M, Park JH, Kato T, Harada M, Matsui S, Ueno M, Fukuda K, Suzuki N, Hazama S, Nagano H, Takeuchi H, Vigneswaran WT, Kitagawa Y and Nakamura Y: TCR sequencing analysis of cancer tissues and tumor draining lymph nodes in colorectal cancer patients. Oncoimmunology 8(6): e1588085, 2019. PMID: 31069156. DOI: 10.1080/2162402X.2019.1588085 
11 Ren L, Matsuda T, Deng B, Kiyotani K, Kato T, Park JH, Seiwert TY, Vokes EE, Agrawal N and Nakamura Y: Similarity and difference in tumor-infiltrating lymphocytes in original tumor tissues and those of in vitro expanded populations in head and neck cancer. Oncotarget 9(3): 3805-3814, 2018. PMID: 29423084. DOI: 10.18632 /oncotarget.23454

12 The Use of the WHO-UMC System for Standardized Case Causality Assessment. World Health Organization (WHO) Uppsala Monitoring Centre. Available at: https://www.who.int/ medicines/areas/quality_safety/safety_efficacy/WHOcausality_asse ssment.pdf [Last accessed on July 27th, 2020]

13 National Cancer Institute. Common Terminology Criteria for Adverse Events (CTCAE). Version 5.0. Available at: https://ctep.cancer.gov/protocolDevelopment/electronic_applicati ons/ctc.htm, 2017 [Last accessed on July 27th, 2020]

14 Eisenhauer EA, Therasse P, Bogaerts J, Schwartz LH, Sargent D, Ford R, Dancey J, Arbuck S, Gwyther S, Mooney M, Rubinstein L, Shankar L, Dodd L, Kaplan R, Lacombe D and Verweij J: New response evaluation criteria in solid tumours: Revised RECIST guideline (version 1.1). Eur J Cancer 45(2): 228-247, 2009. PMID: 19097774. DOI: 10.1016/j.ejca.2008.10.026

15 Gros A, Parkhurst MR, Tran E, Pasetto A, Robbins PF, Ilyas S, Prickett TD, Gartner JJ, Crystal JS, Roberts IM, TrebskaMcGowan K, Wunderlich JR, Yang JC and Rosenberg SA: Prospective identification of neoantigen-specific lymphocytes in the peripheral blood of melanoma patients. Nat Med 22(4): 433438, 2016. PMID: 26901407. DOI: 10.1038/nm.4051
16 Thommen DS, Koelzer VH, Herzig P, Roller A, Trefny M, Dimeloe S, Kiialainen A, Hanhart J, Schill C, Hess C, Savic Prince S, Wiese M, Lardinois D, Ho PC, Klein C, Karanikas V, Mertz KD, Schumacher TN and Zippelius A: A transcriptionally and functionally distinct PD-1(+) CD8(+) T-cell pool with predictive potential in non-small-cell lung cancer treated with PD-1 blockade. Nat Med 24(7): 994-1004, 2018. PMID: 29892065. DOI: $10.1038 / \mathrm{s} 41591-018-0057-\mathrm{Z}$

17 Ribas A and Wolchok JD: Cancer immunotherapy using checkpoint blockade. Science 359(6382): 1350-1355, 2018. PMID: 29567705. DOI: 10.1126/science.aar4060

18 Schadendorf D, Wolchok JD, Hodi FS, Chiarion-Sileni V, Gonzalez R, Rutkowski P, Grob JJ, Cowey CL, Lao CD, Chesney J, Robert C, Grossmann K, McDermott D, Walker D, Bhore R, Larkin J and Postow MA: Efficacy and safety outcomes in patients with advanced melanoma who discontinued treatment with nivolumab and ipilimumab because of adverse events: A pooled analysis of randomized phase II and III trials. J Clin Oncol 35(34): 3807-3814, 2017. PMID: 28841387. DOI: $10.1200 / J C O .2017 .73 .2289$

$19 \mathrm{Fu} \mathrm{C}$ and Jiang A: Dendritic cells and CD8 T-cell immunity in tumor microenvironment. Front Immunol 9: 3059, 2018. PMID: 30619378. DOI: 10.3389/fimmu.2018.03059

Received June 19, 2020

Revised July 28, 2020

Accepted July 30, 2020 